\title{
Predator learning favours mimicry of a less-toxic model in poison frogs
}

\author{
Catherine R. Darst ${ }^{1} \&$ Molly E. Cummings ${ }^{1}$
}

Batesian mimicry_resemblance of a toxic model by an edible mimic-depends on deceiving predators ${ }^{1}$. Mimetic advantage is considered to be dependent on frequency because an increase in mimic abundance leads to breakdown of the warning signal ${ }^{2,3}$. Where multiple toxic species are available, batesian polymorphism $^{4}$ is predicted-that is, mimics diversify to match sympatric models. Despite the prevalence of batesian mimicry in nature ${ }^{5}$, batesian polymorphism is relatively rare $^{6}$. Here we explore a poison-frog mimicry complex comprising two parapatric models and a geographically dimorphic mimic that shows monomorphism where models co-occur. Contrary to classical predictions, our toxicity assays, field observations and spectral reflectances show that mimics resemble the less-toxic and less-abundant model. We examine "stimulus generalization"7 as a mechanism for this nonintuitive result with learning experiments using naive avian predators and live poison frogs. We find that predators differ in avoidance generalization depending on toxicity of the model, conferring greater protection to mimics resembling the lesstoxic model owing to overlap of generalized avoidance curves. Our work supports a mechanism of toxicity-dependent stimulus generalization $^{8}$, revealing an additional solution for batesian mimicry where multiple models coexist.

In batesian mimicry, an edible species co-opts a warning signal from an unpalatable species to gain advantage through predator deception ${ }^{1}$. If batesian mimics are too common, however, this advantage breaks down as predators learn to ignore the warning signal. Where more than one model species is available ${ }^{4}$, diversifying frequency-dependent selection predicts the evolution of polymorphism in which mimics diverge in appearance to resemble sympatric models ${ }^{6,9,10}$. Batesian polymorphism is suggested to distribute warning signal degradation over several defended model species, enabling the mimic to increase in abundance. Reported accounts of such mimetic polymorphism, however, are relatively rare $^{6}$ and unknown in vertebrate mimicry systems ${ }^{11,12}$.

Here we investigate a mimicry system that is inconsistent with the predictions of frequency dependence. We examine a poison-frog mimicry complex composed of two parapatric models and a geographically varying mimic (Fig. 1). The model Ecuadorian poison frogs Epipedobates bilinguis and Epipedobates parvulus share a similar warning signal of a bright red-spotted dorsum but differ in axilla and groin colouration (Fig. 1b). Their phylogenetically distant relative ${ }^{13}$, Allobates zaparo, is geographically dimorphic, matching each warning signal where models are parapatric (Fig. 1b). Where the two models co-occur, however, the mimic resembles only a single model (E. bilinguis; Figs 1 and 2). Here we use spectral reflectances, toxicity assays, field abundance measurements and predator learning experiments to investigate mechanisms that may be contributing to this pattern in nature.

Theoretical and empirical studies predict that coexistence of aposematic models may lead to (1) batesian polymorphism ${ }^{6,9,10}$, (2) evolution of a mimic phenotype intermediate between model species ${ }^{14,15}$, or (3) mimetic resemblance to the most highly abundant and/or noxious model ${ }^{16-19}$. To test batesian mimicry predictions, we quantified patterns of mimicry, abundance, and toxicity of models and mimic in the zone of overlap. We assessed mimicry by degree of overlap between model and mimic using 95\% confidence ellipses

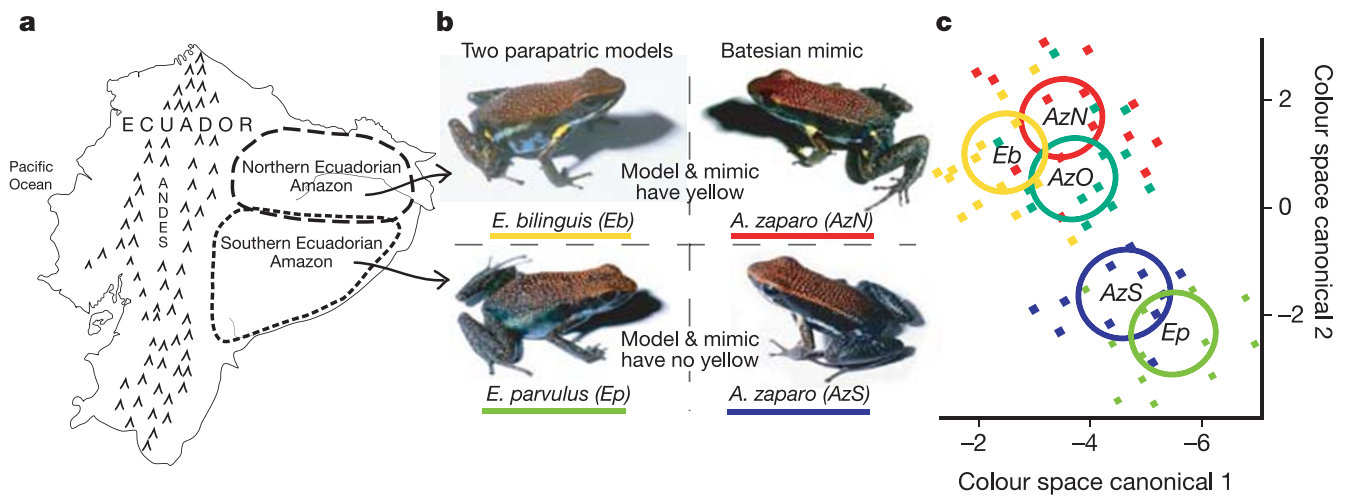

Figure 1 | Poison-frog mimicry complex and colour analyses. a, Geographic distribution of model and mimic species. $\mathbf{b}$, Model and mimic warning signals. c, Discriminate functions plot with colour segments (radiance LS and MUV) ${ }^{20}$ of 'aposematic' frog colours (red, yellow and black) from individuals' head, dorsum, left and right axilla as covariates; and species and locality as categories. Mimicry was determined by overlap of model and mimic $95 \%$ confidence ellipses around the multivariate centroid (Eb: E. bilinguis, $n=25 ; A z N$ : A. zaparo sympatric with $E$. bilinguis in north, $n=15 ; A z O$ : A. zaparo from model species' zone of overlap, $n=13$; AzS: A. zaparo sympatric with $E$. parvulus in south, $n=13$; Ep: E. parvulus, $n=28)$. 

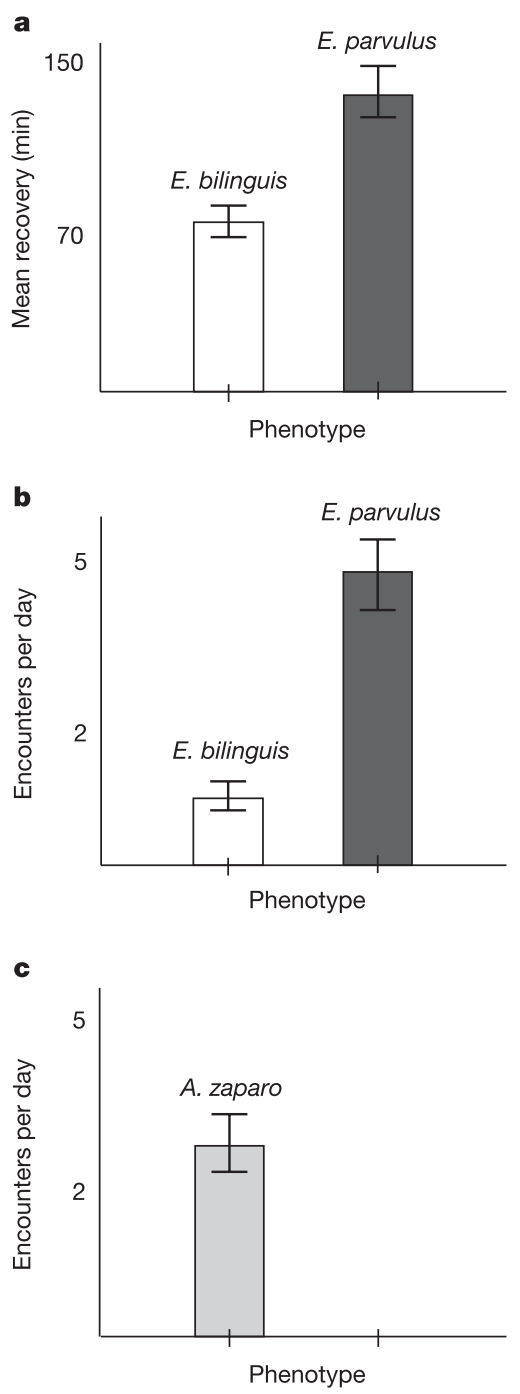

Figure 2 | Measured features of the poison-frog model-mimic system. a, Relative toxicity of models. Shown is the mean recovery time $(\mathrm{min})$ of mice after injection with different model skin extracts. $\mathbf{b}$, Relative abundance of models (b) and mimic phenotype (c) where both models co-occur. Shown is the mean encounter rate per day. Data are mean \pm s.e.m. The mimic in $c$ assumed the E. bilinguis phenotype.

computed from spectral reflectances ${ }^{20}$ (Fig. 1c). The mimic, A. zaparo, shows significant divergence in colour pattern across its geographic range predicted by colour differences between model species (Fig. 1c). Where the two model species co-occur, however, the mimic's warning signal shows significant overlap with only E. bilinguis (Fig. 1c). Thus, in contrast to predictions 1 and 2 for batesian mimics sympatric with multiple models, A. zaparo is neither polymorphic nor intermediate.

Applying prediction 3 to this poison-frog mimicry complex predicts that A. zaparo should mimic the more-toxic and/or abundant model where E. parvulus and E. bilinguis co-occur. To test this prediction, we measured relative abundance as encounter rate across an 8-km transect on 10 consecutive days near the Río Arajuno, Napo Province, Ecuador. We found E. parvulus to be more abundant $(n=43$ in total; mean \pm s.e.m. frogs per day $=4.3 \pm 0.62)$ and E. bilinguis to be less abundant $(n=10$ in total; $1.0 \pm 0.26$ frogs per day; Wilcoxon matched-pairs test, $Z=2.716$; two-tail $P=0.007$; Fig. 2b). We assessed the relative toxicity of the models and mimic using a standard protocol of frog skin extract subcutaneous injection into laboratory mice ${ }^{21}$. The time to recovery from injection of E. parvulus skin extract was significantly greater than the time to

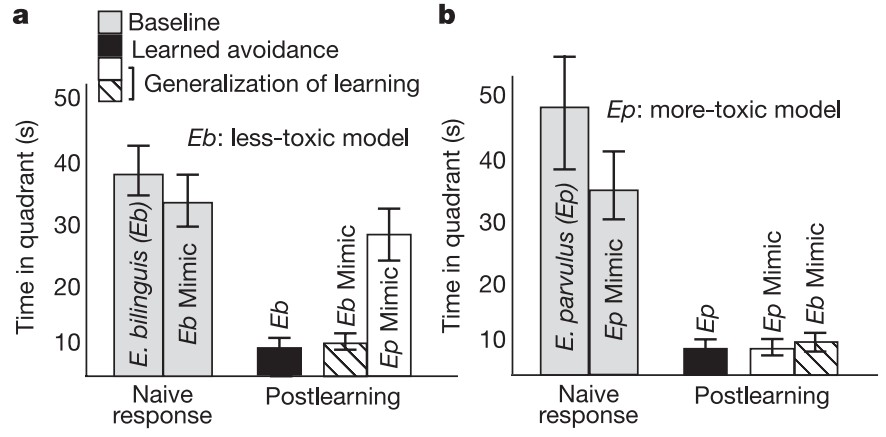

Figure 3 | Predator avoidance learning. Comparison of the chicks' baseline response time with postlearning time (mean \pm s.e.m.) in the frog's test quadrant. a, Less-toxic model, E. bilinguis $(E b)$, as learning stimulus. Chicks learned to avoid $E$. bilinguis (baseline versus postlearning time: $Z=-2.207$, two-tail $P=0.027)$. Learned avoidance generalized to the E. bilinguis mimic A. zaparo north $(Z=-2.201, P=0.028)$, but not to the E. parvulus $(E p)$ mimic $A$. zaparo south $(Z=-0.318, P=0.75)$. b, More-toxic model, E. parvulus, as learning stimulus. Chicks learned to avoid E. parvulus $(Z=-2.201, P=0.028)$. Learned avoidance generalized to the $E$. parvulus mimic $A$. zaparo south $(Z=-2.201, P=0.028)$, and also to the E. bilinguis mimic $A$. zaparo north $(Z=-2.207, P=0.027)$. Data are mean \pm s.e.m.

recovery from injection of $E$. bilinguis skin extract $(n=5$ mice per treatment; mean \pm s.e.m. recovery time: $135.4 \pm 9.31 \mathrm{~min}$ for E. parvulus, $79.0 \pm 3.19 \mathrm{~min}$ for E. bilinguis; $Z=2.023$, two-tail $P=0.043$; Fig. 2a). Injection of A. zaparo skin extract caused no adverse reaction (no difference among reactions from $A$. zaparo skin extract injections and saline control injections: $5.2 \pm 1.8 \mathrm{~min}$ for A. zaparo; $5.1 \pm 1.3 \mathrm{~min}$ for saline control). Thus, in contrast to prediction 3, A. zaparo mimics the less-abundant and less-toxic model, E. bilinguis.

Mimics not only resemble the less-toxic model species in the overlap zone, they also outnumber these models significantly (2.6 \pm 0.50 per day for A. zaparo, $1.0 \pm 0.26$ per day for E. bilinguis; $Z_{n=10}=2.09$; two-tail $P=0.036$; Fig. $2 \mathrm{~b}, \mathrm{c}$ ). To investigate why mimicry of a less-toxic and less-abundant model might be favoured by selection, we conducted predator-learning experiments to explore the classical" tion". Naive chicken predators were exposed to one of the model species in a series of learning trials, and then generalization of learned avoidance was assessed by subsequently exposing the educated predator to the precise mimic phenotype (found in sympatry with the learning stimulus) and the imperfect mimic phenotype (found in sympatry with the other model species). As predicted by singlemodel studies ${ }^{8,17,18}$, we found that predator learning proceeded at a faster rate with the more-toxic model, E. parvulus (mean learning slope: $40.33 \pm 8.11$ for E. parvulus, $18.04 \pm 7.4$ for E. bilinguis; $Z_{n=6}=1.992$, two-tail $\left.P=0.046\right)$. We tested mimic effectiveness (ability to deceive trained predators) and found that predators educated with either model (E. bilinguis or E. parvulus) generalize learned avoidance, on sight, to their respective mimic phenotype of A. zaparo (Fig. 3; mean \pm s.e.m. prelearning and postlearning time in quadrant: $25.83 \pm 4.73 \mathrm{~s}$ and $5.33 \pm 1.05 \mathrm{~s}$, respectively, for E. bilinguis mimic; $25.83 \pm 4.17 \mathrm{~s}$ and $4.17 \pm 1.54 \mathrm{~s}$, respectively, for E. parvulus mimic; $Z_{n=6}=2.201$, two-tail $P=0.028$ ), providing empirical evidence for batesian mimicry in dendrobatid frogs.

We further examined how broadly generalization of avoidance extends or how imperfect a mimic can be and still gain protection from predators educated with a specific model. Although precise mimics enjoyed equal protection regardless of the model species used for learning, imperfect mimics did not. Generalization of learned avoidance to the imperfect mimic differed depending on the toxicity of the model learning stimulus (Fig. 3; mean \pm s.e.m. postlearning time with imperfect mimic: $6.67 \pm 1.05 \mathrm{~s}$ for E. parvulus as learning 

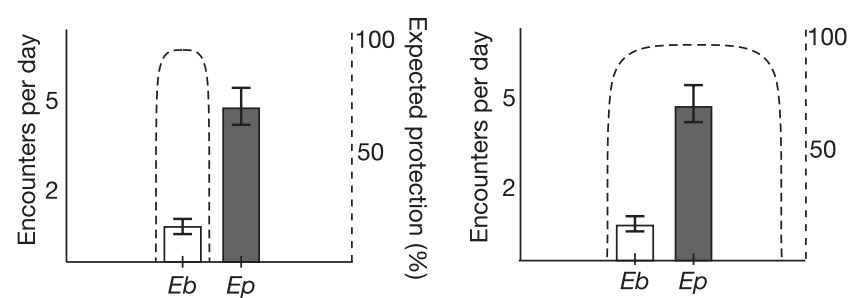

Figure 4 | Generalized avoidance curves. Broken lines represent expected protection for each phenotype estimated using the predator learning data in Fig. 3. Estimates of protection assume fully trained predators in the wild. a, Less-toxic model, E. bilinguis, as learning stimulus. Learned avoidance does not generalize beyond the warning signal with which predators were trained. b, More-toxic model, E. parvulus, as learning stimulus. Learned avoidance generalizes to the mimic of both E. parvulus and E. bilinguis. Thus, A. zaparo individuals resembling less-toxic $E$. bilinguis gain a selective advantage no matter which model is the avoidance learning stimulus. Data are mean \pm s.e.m.

stimulus, $26.67 \pm 4.41 \mathrm{~s}$ for E. bilinguis as learning stimulus; $Z_{n=6}=2.207$; two-tail $\left.P=0.027\right)$. Predators educated with the less-toxic model, E. bilinguis, did not generalize learned avoidance to the mimic of E. parvulus (Fig. 3a; baseline time in quadrant: $28.34 \pm 4.41 \mathrm{~s} ;$ postlearning time with imperfect mimic: $26.67 \pm 4.41 \mathrm{~s} ; Z_{n=6}=0.318$; two-tail $\left.P=0.75\right)$. By contrast, predators educated with the more-toxic model, E. parvulus, did generalize learned avoidance to the imperfect mimic, the mimic of E. bilinguis (Fig. 3b; baseline time: $49.17 \pm 9.17 \mathrm{~s}$; postlearning time with imperfect mimic: $6.67 \pm 1.05 \mathrm{~s} ; Z_{n=6}=2.201$; two-tail $P=0.028$ ). Thus, the stimulus generalization gradient is broader when avoidance is learned on the more-toxic model (avoidance generalizes to both mimic phenotypes) and, in contrast, the stimulus generalization gradient is more narrow when avoidance is learned on the lesstoxic model (avoidance generalizes to only the precise mimic phenotype ${ }^{8,15}$; Fig. 4).

The relative selective advantage gained by either mimic phenotype in the zone of model species overlap is dependent on the penalty to the predator from the particular model being mimicked. Learned avoidance from experience with the more-toxic model will generalize to either mimic phenotype; both mimic phenotypes receive protection if the predator has undergone avoidance learning with moretoxic E. parvulus (Fig. 4b). Learned avoidance from experience with less-toxic E. bilinguis, however, will generalize only to the precise mimic of E. bilinguis (Fig. 4a). In the zone of model species overlap, therefore, mimics of $E$. parvulus only receive protection generated by E. parvulus, whereas mimics of E. bilinguis receive benefits generated by both models.

An alternative explanation for the apparent mimicry mismatch, wherein the mimic resembles the less-toxic and less-abundant model in the overlap zone, may be recent model range expansion (E. parvulus) or contraction (E. bilinguis) in this region. If the range of E. parvulus recently expanded north, or if E. bilinguis populations recently shrank in the overlap zone, then we may be capturing this species complex in an evolutionary lag snapshot-in which the mimic (A. zaparo) has not had enough 'time' to show perfect mimicry to the more-abundant and more-toxic model. Although no range transformation data are available to test this possibility conclusively, it does not rule out the idea that toxicity-dependent generalized avoidance may maintain the current imbalance between mimic and model.

By mimicking the less-toxic model (rather than mimetic polymorphism, an intermediate mimic phenotype, or mimicking the most toxic and/or numerous model), the increased predation risk accrued by an increased abundance of batesian mimic individuals is spread over both defended model species, enabling the mimic to increase in abundance. This non-intuitive result is driven by toxicitydependent generalization of learned avoidance: predators that learn on the more-toxic model will generalize avoidance to the less-toxic model's mimic, whereas predators that learn on the less-toxic model show no generalization beyond this precise warning signal ${ }^{8,15}$. Thus, a mimic of the less-toxic model can enjoy near complete protection from educated predators regardless of which model was used for avoidance learning. We have presented strong evidence suggesting that the selective force influencing A. zaparo's resemblance of the lesstoxic and less-abundant model, E. bilinguis, is stimulus-controlled predator generalization of learned avoidance. Our work therefore provides an adaptive hypothesis based on the classical psychological phenomenon of stimulus generalization ${ }^{7,8}$, which may help to explain the paucity of batesian polymorphism examples, and reveals a monomorphic evolutionary solution to the problem of batesian abundance.

\section{METHODS}

Collection and abundance estimates. Fieldwork was conducted in Amazonian lowland rainforest, between January and May in 2003-2005. In February 2004, we measured poison-frog encounter rates along a $\sim 8-\mathrm{km}$ transect trail for 10 consecutive days in the overlap zone, Río Arajuno $(\sim 3 \mathrm{~km}$ southwest of San Pedro), Napo, Ecuador, of the models. For reflectance measurements and predation experiments, we collected live frogs from Estación Biológica Jatun Sacha, Napo (Allobates zaparo and Epipedobates bilinguis); Río Arajuno, Napo (A. zaparo, E. bilinguis, and E. parvulus); and Santiago, Morona-Santiago (A. zaparo and E. parvulus). For predation experiments, we collected brown, nontoxic Colostethus awa from western Ecuadorian cloudforest at Union del Tuachi, Pichincha. Taxonomy was as described ${ }^{22}$.

Colour analyses. Ninety-four frogs were collected and transported to Museo de Zoología, Universidad Católica del Ecuador (Fig. 1c). Spectral reflectances were measured with an Ocean Optics PS2000 spectrometer, DT-1000 full-spectrum light source, Spectralon white standard and reflectance probe (R400-7) at a 2-mm distance from seven body regions: head, dorsum, axilla, groin, vocal sac, flanks and ventor (two measures per region). We collected leaf-litter background reflectances (Jatun Sacha, six; Río Arajuno, seven; Santiago, seven). Forty-five habitat spectral irradiance measurements were collected at 0900 on $9 \mathrm{~d}$ with the PS2000 and cosine collector. Frog and background radiance estimates were computed as the product of spectral reflectances and average habitat irradiance spectrum.

To compare radiance measurements, independent of the visual system, we used a segments classification method ${ }^{20}$. Radiance spectra were divided into four bandwidths (ultraviolet, 300-399 nm; short, 400-499 nm; middle, 500-599 nm; long, 600-699 nm), normalized by total intensity, and evaluated in a twodimensional space by orthogonal axes representing hypothetical opponency processes (LS, long-short; MUV, middle-ultraviolet). We computed composite euclidean distances ${ }^{20}, D_{\text {comp }}$, representing distance in colour space between frog and leaf-litter background. Whole-body colouration measures were similar between model species $\left(D_{\text {comp: }}: 22.18 \pm 3.88\right.$ for E. parvulus, $20.24 \pm 5.30$ for E. bilinguis; $t=0.456$, two-tail $P=0.664)$. To evaluate mimicry, we used multivariate discriminate functions analyses of warning coloured segments in $\mathrm{JMP}^{23,24}$ (Fig. 1c).

Toxicity assays. Five frogs from each species were killed and skinned as described $^{25}$. Methanol extracts from individual frogs were evaporated and resuspended in sterile saline. Resultant single-skin extracts were subcutaneously injected in four treatments, each given to five mice ${ }^{21}(n=20$ mice, IACUC 03110501), as follows: E. bilinguis, E. parvulus, A. zaparo or saline control. Sleeping behaviour was the baseline for toxicity assays. Mice were awakened with the injection and the time to complete recovery (return to sleep) was recorded. Recovery time was used to estimate the degree of toxicity.

Predator learning experiments. Although few data exist, birds may be potential predators of poison frogs ${ }^{26}$. Thus, in Ecuador we conducted a series of learning experiments using $\sim 1$-month-old domestic chickens (Gallus gallus domesticus) as naive, model predators ${ }^{27}$ and wild-caught dendrobatid frogs (models, E. bilinguis and E. parvulus; mimic, A. zaparo). Birds were tested individually in a $1-\mathrm{m}^{2}$ dirt-floor test arena of four $50-\mathrm{cm}^{2}$ quadrants, outside under natural lighting conditions. Chickens were fed chicken mash and cracked corn twice daily and water ad libitum. We assessed mimic palatability by presenting six naive chickens with an A. zaparo (three northern and three southern A. zaparo). Naive chickens readily ate both $A$. zaparo and control frogs (C. awa).

We had two experimental groups ( six chicks each), which differed in learning 
stimulus (E. parvulus or E. bilinguis) in eight learning trails (IACUC 04071901). Learning trials consisted of presenting a chick with a learning stimulus under a glass dome for $1 \mathrm{~min}$ or until the chick pecked the dome. The dome was then removed and latency to approach the stimulus was recorded for up to $2 \mathrm{~min}$ or until a sampling event. In a typical sampling event, chicks grabbed the frog in their beaks and spat the frog out. Only one chick ingested a poison frog (E. bilinguis); it died $3 \mathrm{~d}$ later and its data were removed. All other chicks tasted and released the frog; most frogs survived the sampling event. We defined the learning rate as the slope (latency to peck divided by number of trials) until complete avoidance (no subsequent sampling in further trials). Control frogs were presented to chicks after trials 2 and 6 to assure that chicks were still motivated to eat frogs.

After training was complete, learning and learning generalization were assessed in choice experiments that paired the control frog with one of three brightly coloured dendrobatid frogs: toxic model learning stimulus (learned avoidance); precise mimic of learning stimulus (learning generalization); and imperfect mimic of learning stimulus (degree of generalization). Chicks were presented with both the brightly coloured frog and a control frog each under a glass dome for $2 \mathrm{~min}$, and the time spent in the test arena quadrant of each dome was recorded. Frog placement in the test arena was randomized across trials. We assessed learned avoidance and generalization of learned avoidance by comparing prelearning (baseline) and postlearning time spent by chicks in the brightly coloured frog's test arena.

\section{Received 31 August; accepted 4 October 2005.}

1. Bates, H. W. Contributions to an insect fauna of the Amazon valley. Lepidoptera: Heliconidae. Trans. Linn. Soc. Lond. 23, 495-566 (1862)

2. Fisher, R. A. Genetical Theory of Natural Selection (Clarendon, Oxford, 1930).

3. Brower, L. P. \& Brower, J. V. Z. The relative abundance of model and mimic butterflies in natural populations of the Battus philenor mimicry complex. Ecology 43, 154-158 (1962).

4. Wallace, A. R. On the phenomena of variation and geographical distribution as illustrated by the Papilionidae of the Malayan region. Trans. Linn. Soc. Lond. 25, $1-71$ (1865).

5. Edmunds, M. Defence in Animals (Longman, Harlow, 1974).

6. Joron, M. \& Mallet, J. L. B. Diversity in mimicry: paradox or paradigm? Trends Ecol. Evol. 13, 461-466 (1998).

7. Pavlov, I. P. Conditioned Reflexes (translated by Anrep, G. V.) (Univ. Press, London, 1927)

8. Duncan, C. J. \& Sheppard, P. M. Sensory discrimination and its role in the evolution of Batesian mimicry. Behaviour 24, 269-282 (1965).

9. Ford, E. B. Ecological Genetics 3rd edn (Chapman and Hall, London, 1971).

10. Turner, J. R. G. The evolutionary dynamics of Batesian and Müllerian mimicry: similarities and differences. Ecol. Entomol. 12, 81-95 (1987).

11. Brodie, E. D. Jr \& Brodie, E. D. III Differential avoidance of mimetic salamanders by free-ranging birds. Science 208, 181-182 (1980).
12. Greene, H. W. \& McDiarmid, R. W. Coral snake mimicry: does it occur? Science 213, 1207-1212 (1981).

13. Santos, J. C., Coloma, L. A. \& Cannatella, D. C. Multiple, recurring origins of aposematism and diet specialization in poison frogs. Proc. Natl Acad. Sci. USA 100, 12792-12797 (2003).

14. Edmunds, M. Why are there good mimics and poor mimics? Biol. J. Linn. Soc. 70, 459-466 (2000).

15. Sherratt, T. N. The evolution of imperfect mimicry. Behav. Ecol. 13, 821-826 (2002).

16. Brower, J. V. Z. Experimental studies of mimicry. IV. The reactions of starlings to the different proportions of models and mimics. Am. Nat. 94, 271-282 (1960).

17. Goodale, M. A. \& Sneddon, I. The effect of distastefulness of the model on the predation of artificial Batesian mimics. Anim. Behav. 25, 660-665 (1977).

18. Lindström, L., Alatalo, R. V. \& Mappes, J. Imperfect Batesian mimicry-the effects of the frequency and the distastefulness of the model. Proc. R. Soc. Lond. B 264, 149-153 (1997).

19. Johnstone, R. A. The evolution of inaccurate mimics. Nature $418,524-526$ (2002).

20. Endler, J. A. On the measurement and classification of colour in studies of animal colour patterns. Biol. J. Linn. Soc. 41, 315-352 (1990).

21. Daly, J. W., Garraffo, H. M. \& Spande, T. F. in Alkaloids: Chemical and Biological Perspectives vol. 13 (ed. Pelletier, S. W.) 1-161 (Pergamon, New York, 1999)

22. Frost, D. R. Amphibian Species of the World 3.0: an Online Reference 〈http:// research.amnh.org/herpetology/amphibia/index.html〉 (American Museum of Natural History, New York, 2004).

23. SAS Institute. JMP Statistics and Graphics Guide version 4 (SAS Institute, Cary, NC, 2000).

24. Sokal, R. R. \& Rohlf, F. J. Biometry: the Principles and Practice of Statistics in Biological Research 2nd edn (W. H. Freeman and Co., San Francisco, 1981).

25. Darst, C. R., Menéndez-Guerrero, P. A., Coloma, L. A. \& Cannatella, D. C. Evolution of dietary specialization and chemical defense in poison frogs (Dendrobatidae): a comparative analysis. Am. Nat. 165, 56-69 (2005).

26. Master, T. L. Dendrobates auratus (black and green poison dart frog): predation. Herp. Rev. 29, 164-165 (1998)

27. Osorio, D., Vorobyev, M. \& Jones, C. D. Colour vision of domestic chicks. J. Exp. Biol. 202, 2951-2959 (1999).

Acknowledgements We thank L. Coloma, J. C. Santos, and S. Ron for discussions on poison frogs; D. Cannatella for frog photos in Fig. 1, assistance with assays and discussion; $M$. Domjan for advice on predator learning experiments; E. Tapia, S. Padilla, M. Bustamante, P. Menéndez-Guerrero and D. Paucar for assistance in the field; J. Cassaday for assistance with toxicity assays; and M. Ryan for comments on the manuscript. This work was supported by University of Texas at Austin EEB graduate program fellowships, a UT Continuing Fellowship, and the Explorer's Club Exploration Fund.

Author Information Reprints and permissions information is available at npg.nature.com/reprintsandpermissions. The authors declare no competing financial interests. Correspondence and requests for materials should be addressed to C.R.D. (catdarst@mail.utexas.edu). 